\title{
Considerations and Pitfalls in Selecting the Drug Vehicles for Evaluation of New Drug Candidates: Focus on in vivo Pharmaco-Toxicological Assays Based on the Rotarod Performance Test
}

\author{
Mariana Matias $^{\mathrm{a}, \mathrm{b}}$, Samuel Silvestre ${ }^{\mathrm{a}, \mathrm{b}}$, Amílcar Falcão $^{\mathrm{b}, \mathrm{c}}$, Gilberto Alves ${ }^{\mathrm{a}, \mathrm{b}}$ \\ ${ }^{a}$ CICS-UBI - Health Sciences Research Centre, University of Beira Interior, Covilhã, Portugal; ${ }^{\mathrm{b}} \mathrm{CNC}-\mathrm{Center}$ for \\ Neuroscience and Cell Biology, University of Coimbra, Coimbra, Portugal; ' Pharmacology Department, Faculty of \\ Pharmacy, University of Coimbra, Pólo das Ciências da Saúde, Azinhaga de Santa Comba, Coimbra, Portugal
}

Received, December 8, 2017; Revised, March 8, 2018; Accepted, March 9, 2017; Published, March 13, 2018.

\begin{abstract}
Purpose - During the discovery and development of new drugs, compounds with low aqueous solubility pose special challenges in their pharmacological evaluation and, therefore, the selection of appropriate vehicles to administer the compounds of interest is determinant for the quality of the results generated during the in vivo non-clinical studies. This work aimed to evaluate the motor deficit (as a surrogate of neurotoxicity) of several administration/delivery vehicles through the rotarod performance test. Methods Trained male CD-1 mice were intraperitoneally administered with the following vehicles: dimethyl sulfoxide (DMSO), aqueous sodium chloride $(\mathrm{NaCl}) 0.9 \%$, aqueous carboxymethylcellulose $(\mathrm{CMC}) 0.5 \%$, polyethylene glycol (PEG)-400, propylene glycol (PG), and solutions of these vehicles containing 5\% and $10 \%$ DMSO. Results - It was observed that the aqueous vehicles $(\mathrm{NaCl} 0.9 \%$ and $\mathrm{CMC} 0.5 \%)$ did not affect the performance of the animals on the rod. On the other hand, a vehicle consisting solely of DMSO led to significant motor impairment and only a small improvement was recorded over time. Additionally, a strong neuromotor toxicity was observed in the early evaluation points of the experiment using vehicles constituted by PG and PEG-400 or by mixtures of PG/DMSO (5\% and 10\%) and PEG-400/DMSO (5\% and 10\%). Conclusion - This study provides useful data about the neurotoxicity inherent to several vehicles frequently used in non-clinical pharmaco-toxicological assays, aiming to draw especial attention to the need of a careful selection of drug vehicles in order to avoid the impact of such confounding variables on the accuracy of the results and in decision-making processes.
\end{abstract}

This article is open to POST-PUBLICATION REVIEW. Registered readers (see "For Readers") may comment by clicking on ABSTRACT on the issue's contents page.

\section{INTRODUCTION}

In the discovery and development of new drug candidates, the solubility of the test compounds is one of the physicochemical properties that must be considered and assessed since the early stages of drug research (1). Indeed, nowadays, it is widely accepted by the scientific community that the solubility of the drug compounds, especially its aqueous solubility, is a major indicator for the drug dissolution in physiological fluids, which is the limiting step for drug absorption and consequently to achieve the pharmacological activity $(2,3)$. In fact, even for first in vivo preclinical screening studies, a suitable formulation strategy is required in order to enable an appropriate administration of the test compounds with acceptable tolerability and maintaining the stability for a sufficient period of time with no adverse effects in animal tests that could be attributed to the delivery vehicles $(4,5)$. Particularly, when the compound of interest is developed to act in the central nervous system
(CNS), its solubility is a very relevant challenge because, usually, is necessary a considerable degree of lipophilicity to cross the blood-brain barrier.

In this context, whenever possible, the choice of the delivery/administration vehicle falls in isotonic physiological saline solutions, which are considered innocuous. However, commonly, the test compounds are not soluble in this type of aqueous solvents due to their intrinsic lipophilicity and other options have to be considered. Dimethyl sulfoxide (DMSO), a polar organic solvent, often emerges as a relevant alternative, and many studies about its pharmacological and toxicological effects have been carried out over the years (6-8).

\footnotetext{
Corresponding Author: Gilberto Alves, PharmD, $\mathrm{PhD}$, Faculty of Health Sciences, CICS-UBI - Health Sciences Research Centre, University of Beira Interior; Av. Infante D. Henrique, 6200-506 Covilhã, Portugal; E-mail address: gilberto@fcsaude.ubi.pt
} 
Indeed, DMSO has been frequently included in different percentages in administration vehicles of compounds tested in whole-animal assays (9-11). Other examples of delivery vehicles widely used by the pharmaceutical industry in different formulations are propylene glycol (PG) (12) and polyethylene glycol (PEG) $(13,14)$, which have the advantages of being soluble in polar and non-polar solvents and are quite inexpensive. In addition, they have been considered non-toxic $(15,16)$. Furthermore, carboxymethylcellulose (CMC) is one of the most commonly used biopolymers in biomedical applications because it is also considered environmentally friendly and non-toxic (17-19).

The preclinical assessment of the "minimal neurological deficit" in rodents (mice and rats) is an essential task in primary and secondary pharmacological screening either in the early stages of drug development of new CNS-active drugs to screen out less promising compounds (2024) or during the safety evaluation of peripheralacting drugs in order to investigate adverse/toxic effects that could cause impairments later (25-27). Overall, the rotarod performance test has been widely used to indirectly assess the minimal neurological deficit in rodents induced by test compounds through the evaluation of the impairment of functions as balance and/or motor coordination. This behavioral assay has gained increasing importance in the discovery and development programs of new drug candidates as it is very simple to perform and allows the evaluation of a large set of compounds. Furthermore, the rotarod performance test is a versatile whole-animal assay that can be used for the assessment of any new molecular entity, regardless of its therapeutic area (28).

Over the last years our research group has been focused on the development of new antiepileptic drug candidates (29-34) and the selection of the administration/delivery vehicle to be employed for solubilization/suspension of test compounds continues to be a challenge in order to appropriately conduct pharmacokinetics and/or pharmaco-toxicological experiments in in vivo conditions. Hence, an analysis of the literature concerning the solvents and/or mixtures of solvents that are used to evaluate potential anticonvulsant compounds in gold standard assays of efficacy (maximal electroshock seizure test and subcutaneous pentylenetetrazole assay) and toxicity (rotarod test) $(11,13,35,36)$ revealed that the impact of the administration vehicles on the obtained results has not been clearly evaluated and discussed. Although the influence of the delivery vehicle may be negligible in many pharmacotoxicological assays, we suspected that this might not be the case in more sensitive behavioral assays, such as the rotarod performance test. Thus, the aim of the present study was to assess the minimal neuromotor impairment (neurotoxicity) induced by a set of the most common vehicles and their mixtures using the rotarod performance test.

\section{MATERIAL AND METHODS}

\section{Chemicals and reagents}

DMSO, CMC sodium and PG were obtained from Sigma (St. Louis, MO, USA). Sodium chloride $(\mathrm{NaCl}) \quad 0.9 \%$ was obtained from B. Braun (Bethlehem, PA, USA). PEG-400 was obtained from Merck Schuchardt (Hohenbrunn, Germany).

\section{Animals}

Adult male CD-1 mice, aged between 6-7 weeks, were obtained from local certified animal facilities (Faculty of Health Sciences of the University of Beira Interior, Covilhã, Portugal). Four mice per cage were housed under controlled environmental conditions [12 h light/dark cycle (lights on at 8:00 $\mathrm{AM})$ at $20 \pm 2{ }^{\circ} \mathrm{C}$ and relative humidity $50 \pm 5 \%$ ] with free access to tap water and standard rodent diet (4RF21, Mucedola, Italy). All experimental and care procedures were conducted in accordance with the European Directive (2010/63/EU) regarding the protection of laboratory animals used for scientific purposes.

\section{Administration/delivery vehicles}

Mice were intraperitoneally injected with each delivery/administration vehicle $(10 \mu \mathrm{L} / \mathrm{g}$ of body weight). The vehicles assessed included the individual solvents (DMSO, $\mathrm{NaCl} 0.9 \%, \mathrm{CMC}$ $0.5 \%$, PEG-400 and PG) and also solutions of $\mathrm{NaCl} 0.9 \%$, CMC 0.5\%, PEG-400 and PG containing $5 \%$ and $10 \%$ DMSO.

\section{Minimal motor impairment (rotarod) test}

Minimal motor impairment was evaluated in mice by standard rotarod performance test as previously reported (37). Mice were previously trained to balance on an accelerating rotarod apparatus (rod diameter: $3 \mathrm{~cm}$ ) that rotated at a constant speed of 10 revolutions per minute (Rota-rod, Ugo Basile, Varese, Italy). During the training sessions, the animals were placed on the rotating rod at least three consecutive trials for $90 \mathrm{~s}$. On the day of the test, trained mice were injected with each delivery/administration vehicle and the motor/neurological toxicity was indicated by the inability of the animal to maintain equilibration on 
the rod for at least $60 \mathrm{~s}$ (primary endpoint). The mice were placed on the rod at predefined timepoints $(0.5 \mathrm{~h}, 1 \mathrm{~h}, 1.5 \mathrm{~h}, 2 \mathrm{~h}, 2.5 \mathrm{~h}, 3 \mathrm{~h}, 3.5 \mathrm{~h}$ and 4 h) after the administration of each vehicle and fall off time was recorded ( $n=4$ per group). In this assay the number of animals that performed the test with success (primary endpoint) was recorded and, additionally, the number of seconds that each animal remained on the rod was also registered (secondary endpoint).

\section{STATISTICAL ANALYSIS}

Data were reported as the mean \pm standard error of the mean. Comparison among groups was analyzed by using the one-way ANOVA with the post hoc Dunnett's multiple comparison test to judge significance of the observed effects. Differences were considered to be statistically significant for a $p$-value lower than $0.05(p<0.05)$.

\section{RESULTS AND DISCUSSION}

In this study, different administration vehicles were evaluated, which were chosen based on their potential usefulness for the solubilization/suspension and delivery of drug candidates in the first steps of non-clinical in vivo pharmaco-toxicological assays. The evaluation of the neurotoxic effects of the test set of administration vehicles was performed by means of the rotarod assay. This experimental evaluation has proved to have a remarkable value in the screening of potential side effects of drugs or drug candidates in the CNS, which are manifested on the balance and motor coordination required to successfully achieve the primary endpoint of the assay. In this comparative study we evaluated not only the potential neurotoxicity of the vehicles themselves ( $\mathrm{NaCl} 0.9 \%$, DMSO, PEG-400, PG and CMC $0.5 \%$ ) but also solutions of these vehicles with different percentages of DMSO $(5 \%$ and $10 \%)$.

Interestingly, it was observed that all vehicles containing $\mathrm{NaCl} 0.9 \%$ and $\mathrm{CMC} 0.5 \%$ with or without DMSO did not produce any motor impairment (Table 1). Probably this occurred due to the fact that these vehicles consisted in aqueous solvents and, therefore, they seem to be the safest option whenever they can be considered. Particularly, the vehicles containing CMC $0.5 \%$ with a small percentage of DMSO may be a good option to administer compounds that show poor solubility in $\mathrm{NaCl} 0.9 \%$ solutions. Indeed, several strategies have been developed to improve the solubility of compounds in this type of vehicles during the optimization phase of drug discovery and development. These include in silico methodologies to predict drug solubility, chemical modifications involving the preparation of prodrugs or the introduction of adequate functional groups, use of co-solvents and developing formulations to increase solubility and dissolution rate (38-40).

Regarding the discovery and development of new drug candidates, DMSO has become the solvent of choice to dissolve potential neuroprotective and neurotoxic hydrophobic substances used in pharmaco-toxicological research $(41,42)$ that generally do not solubilize in aqueous vehicles. Some DMSO advantages include its capacity to dissolve compounds with a wide range of chemical properties, low volatility, miscibility with water, relatively low toxicity to both tissue culture and technicians, and limited deleterious effects at low concentration upon bioassays $(43,44)$. In addition, it has been suggested that DMSO can be safely used, being generally well-tolerated by the experimental animals $(45,46)$ and itself may still have benefits to specific disorders $(47,48)$. However, there are also several case reports revealing severe neurotoxicity associated with DMSO, indicating the importance of its careful use in certain circumstances, including as administration vehicle, to avoid confounding factors that can bias the study results and lead to seriously erroneous conclusions $(41,49,50)$. Because of these contradictions and the established importance of DMSO as drug vehicle, it was our interest to evaluate its neurological toxicity in in vivo non-clinical studies using the rotarod performance test. In fact, it was verified in the present study that the administration of a vehicle $(10 \mu \mathrm{L} / \mathrm{g})$ consisting of $100 \%$ DMSO originates motor impairment (a surrogate of neurotoxicity or minimal neurological deficit) on the rotarod assay, with animals falling from the rod at all post-dose time-points considered in the study (Table 1). In addition, it was observed that, at the first time-points, the animals receiving vehicles (10 $\mu \mathrm{L} / \mathrm{g}$ ) containing PEG-400 showed a notable toxicity, but at $4 \mathrm{~h}$ after the intraperitoneal injection, $100 \%$ of the animals successfully reached the primary endpoint of the assay. On the other hand, until $2.5 \mathrm{~h}$ after the injection of the vehicles containing PG, $75-100 \%$ of the mice showed inability to maintain equilibration on the rod for at least $60 \mathrm{~s}$ (primary endpoint). Moreover, considering the evaluation data for $\mathrm{PG}$, it was verified that the animals receiving this delivery vehicle containing $10 \%$ of DMSO recovered the motor coordination faster than the animals 
receiving only $\mathrm{PG}$ or $\mathrm{PG}$ with $5 \%$ of DMSO (neurotoxicity in $25 \%$ versus $75-100 \%$ of the animals at $4 \mathrm{~h}$ ).

Besides the number of animals that successfully achieved the primary endpoint of the rotarod performance test (maintaining equilibrium on the rod for at least $60 \mathrm{~s}$ ), it was also recorded the exact time in seconds (secondary endpoint) that each animal remained on the rod during the time set for the test. The results are illustrated in Figure 1. As can be seen, at the first time-points of the study it is possible to notice that the toxicity triggered by pure DMSO was lower than the observed with PEG-400 alone $(1 \mathrm{~h})$ and with vehicles containing PG.

Concerning the reasons explaining the DMSOinduced neurotoxicity, some evidence suggests that its mechanisms of toxicity can involve neurophysiological and pathological changes including myelin disruption and uncompacted myelin lamella (49). In addition, DMSO could also be able to produce widespread, dose-dependent neurodegeneration in the developing mouse brain at several ages and this toxicity probably results from a direct cellular effect. Furthermore, the DMSO-induced apoptosis might produce significant learning and memory deficits (50). Additional evidence has indicated that glutamate receptors could be involved in the neurotoxicity originated by DMSO (48); however, its exact mechanisms of toxicity remains unknown. Moreover, these studies just had focus on chronic neurotoxicity exhibited by DMSO and they probably could not explain the acute toxicity observed in our research.

In the present study it was also observed that all PG solutions possibly reached the brain rapidly because a strong neuromotor impairment in the rotarod performance test was noted. Particularly, the vehicles consisting of PG $(100 \%)$ and $\mathrm{PG} / \mathrm{DMSO}(95 \% / 5 \%, v / v)$ in all steps of the study led to higher neuromotor impairment than the observed with $100 \%$ DMSO. On the other hand, although the PG/DMSO $(90 \% / 10 \%, v / v)$ solution originated evident neuromotor deficit in the early steps of the study, the recovery of the animals was faster than those receiving DMSO and the other vehicles containing PG. As demonstrated for DMSO, PG has also been suggested to produce apoptotic neurodegeneration in a dose-dependent manner. Furthermore, the observed damage was dependent on age at the time of exposure and probably PG does not produce damage through GABA receptors. It is still unknown whether apoptosis results in long-term cognitive and behavioral abnormalities (51).

After the administration of PEG-400containing vehicles, interestingly, it was verified that with the increase of the DMSO percentage in the vehicle, the neuromotor toxicity seems to be reduced. In fact, 3 hours after the administration of PEG-400 with the highest percentage of DMSO $(10 \%)$ all the animals performed the test without any evident neuromotor deficit. Actually, DMSO was reported as having anti-nociceptive and antiinflammatory effects in male CD-1 mice when given orally $(10 \mathrm{~mL} / \mathrm{kg})$ or by intracerebroventricular route (5 $\mu \mathrm{L} /$ mouse) (52), which could be a possible explanation for our results.

Regarding the PG-containing vehicles, another possible explanation for the neuromotor toxicity observed could be associated with the hyperosmolality effects and increase of the anion

Table 1. Time-course of minimal neurological impairment (neurotoxicity) of vehicles administered intraperitoneally to mice in the rotarod performance test (number of animals exhibiting neurological impairment/number of animals tested).

\begin{tabular}{|c|c|c|c|c|c|c|c|c|}
\hline Vehicle & $0.5 \mathrm{~h}$ & $1 \mathrm{~h}$ & $1.5 \mathrm{~h}$ & $2 \mathrm{~h}$ & $2.5 \mathrm{~h}$ & $3 \mathrm{~h}$ & $3.5 \mathrm{~h}$ & $4 \mathrm{~h}$ \\
\hline DMSO & $4 / 4$ & $4 / 4$ & $4 / 4$ & $4 / 4$ & $4 / 4$ & $3 / 4$ & $3 / 4$ & $3 / 4$ \\
\hline $\mathrm{NaCl} 0.9 \%$ & $0 / 4$ & $0 / 4$ & $0 / 4$ & $0 / 4$ & $0 / 4$ & $0 / 4$ & $0 / 4$ & $0 / 4$ \\
\hline $\mathrm{NaCl} 0.9 \% / \mathrm{DMSO}(95 \% / 5 \%, v / v)$ & $0 / 4$ & $0 / 4$ & $0 / 4$ & $0 / 4$ & $0 / 4$ & $0 / 4$ & $0 / 4$ & $0 / 4$ \\
\hline $\mathrm{NaCl} 0.9 \% / \mathrm{DMSO}(90 \% / 10 \%, v / v)$ & $0 / 4$ & $0 / 4$ & $0 / 4$ & $0 / 4$ & $0 / 4$ & $0 / 4$ & $0 / 4$ & $0 / 4$ \\
\hline PEG-400 & $4 / 4$ & $4 / 4$ & $4 / 4$ & $3 / 4$ & $2 / 4$ & $1 / 4$ & $1 / 4$ & $0 / 4$ \\
\hline PEG-400/DMSO $(95 \% / 5 \%, v / v)$ & $4 / 4$ & $3 / 4$ & $3 / 4$ & $3 / 4$ & $1 / 4$ & $0 / 4$ & $0 / 4$ & $0 / 4$ \\
\hline PEG-400/DMSO $(90 \% / 10 \%, v / v)$ & $4 / 4$ & $3 / 4$ & $2 / 4$ & $2 / 4$ & $2 / 4$ & $0 / 4$ & $0 / 4$ & $0 / 4$ \\
\hline $\mathrm{PG}$ & $4 / 4$ & $4 / 4$ & $4 / 4$ & $4 / 4$ & $4 / 4$ & $4 / 4$ & $4 / 4$ & $3 / 4$ \\
\hline PG/DMSO $(95 \% / 5 \%, v / v)$ & $4 / 4$ & $4 / 4$ & $4 / 4$ & $4 / 4$ & $4 / 4$ & $4 / 4$ & $4 / 4$ & $4 / 4$ \\
\hline PG/DMSO $(90 \% / 10 \%, v / v)$ & $4 / 4$ & $4 / 4$ & $3 / 4$ & $3 / 4$ & $3 / 4$ & $2 / 4$ & $1 / 4$ & $1 / 4$ \\
\hline CMC $0.5 \%$ & $0 / 4$ & $0 / 4$ & $0 / 4$ & $0 / 4$ & $0 / 4$ & $0 / 4$ & $0 / 4$ & $0 / 4$ \\
\hline CMC $0.5 \% / \mathrm{DMSO}(95 \% / 5 \%, v / v)$ & $0 / 4$ & $0 / 4$ & $0 / 4$ & $0 / 4$ & $0 / 4$ & $0 / 4$ & $0 / 4$ & $0 / 4$ \\
\hline CMC $0.5 \% / \mathrm{DMSO}(90 \% / 10 \%, v / v)$ & $0 / 4$ & $0 / 4$ & $0 / 4$ & $0 / 4$ & $0 / 4$ & $0 / 4$ & $0 / 4$ & $0 / 4$ \\
\hline
\end{tabular}

$\mathrm{CMC}$, carboxymethylcellulose; DMSO, dimethyl sulfoxide; $\mathrm{NaCl}$, sodium chloride; $\mathrm{PEG}-400$, polyethylene glycol-400; PG, propylene glycol. 
$0.5 \mathrm{~h}$

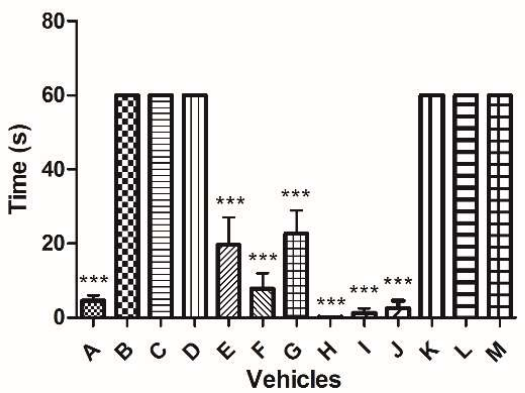

$1.5 \mathrm{~h}$

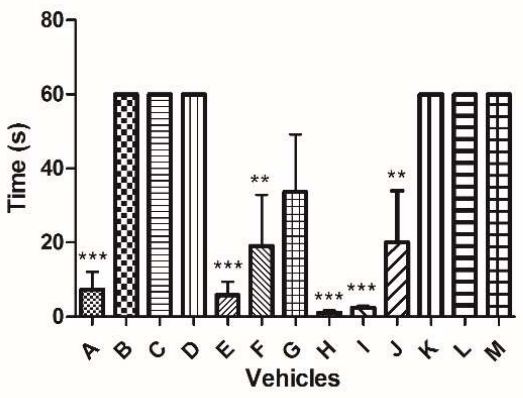

$2.5 \mathrm{~h}$

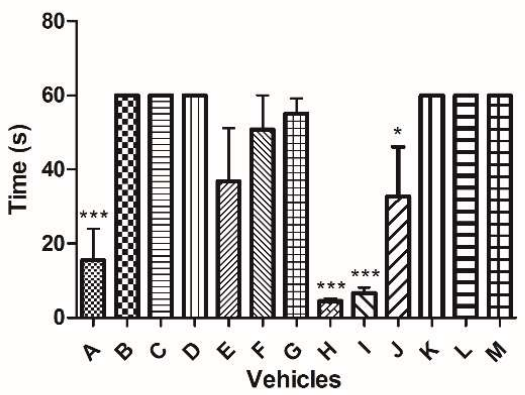

$3.5 \mathrm{~h}$

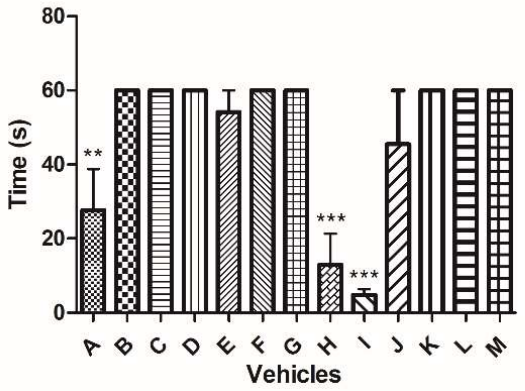

$1 \mathrm{~h}$

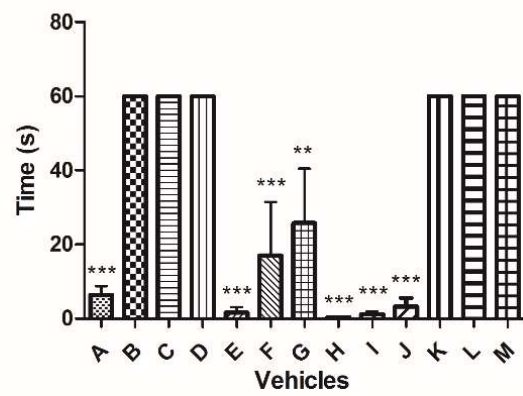

$2 \mathrm{~h}$

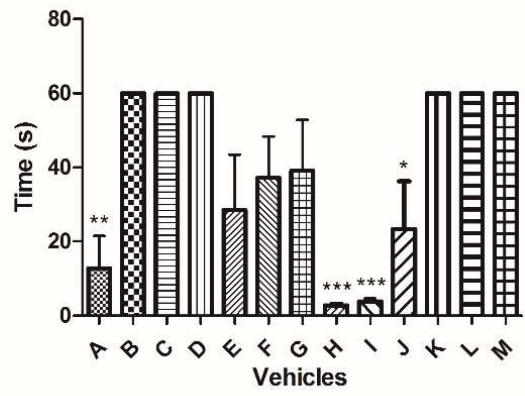

$3 \mathrm{~h}$

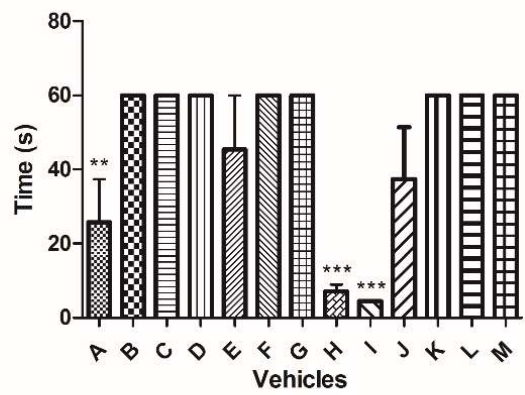

$4 \mathrm{~h}$

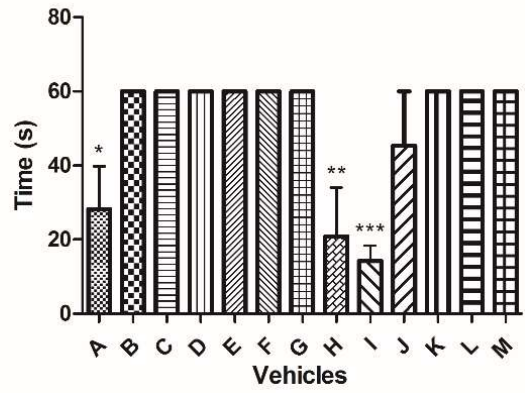

Figure 1 - Effects induced by commonly used drug vehicles on the time (in seconds) that intraperitoneally administered mice $(n=4)$ remained on the rod during the rotarod performance test. The test was performed at several predefined postdose time-points $(0.5 \mathrm{~h}, 1 \mathrm{~h}, 1.5 \mathrm{~h}, 2 \mathrm{~h}, 2.5 \mathrm{~h}, 3 \mathrm{~h}, 3.5 \mathrm{~h}$ and $4 \mathrm{~h})$. A - Dimethyl sulfoxide (DMSO), B - Aqueous sodium chloride $(\mathrm{NaCl})$ 0.9\%, C - NaCl 0.9\%/DMSO $(95 \% / 5 \%, v / v), \mathbf{D}-\mathrm{NaCl}$ 0.9\%/DMSO (90\%/10\%, v/v), E - Polyethylene glycol (PEG)-400, F - PEG-400/DMSO (95\%/5\%, v/v), G - PEG-400/DMSO (90\%/10\%, v/v), H - Propylene glycol (PG), I - PG/DMSO (95\%/5\%, v/v), J - PG/DMSO (90\%/10\%, v/v), K - Aqueous carboxymethylcellulose (CMC) $0.5 \%$, $\mathbf{L}-$ CMC 0.5\%/DMSO (95\%/5\%, v/v), M - CMC 0.5\%/DMSO $(90 \% / 10 \%, v / v) .{ }^{*} p<0.05, * * p<0.01, * * * p<0.001$ compared to control group (B). 
gap metabolic acidosis (due to lactic acidosis) that was observed in humans. For instance, after an injection of lorazepam was observed that PG had a much greater contribution than PEG for the hyperosmolar metabolic acidosis (53). This information can be useful to understand the differences between PG and PEG-400 in our results.

Moreover, it should be highlighted that after the injections of PEG-400, PG and DMSO (100\%) hypoactivity and immobility was noticed in the animals, which was consequently expressed in the performance on the rod. These results were in accordance with the observations of a previous study which analyzed several solvents administered intravenously in female CD-1 mice, which aimed to understand the tolerability and recommended solvent dose limits for pharmacokinetic studies (54).

Independently of the causes underlying the neuromotor toxicity observed with these delivery vehicles, this study shows that their use to evaluate the neurotoxicity of new drug candidates through the rotarod assay can be debatable. In fact, during the revision of literature it was frequently found the usage of PEG-400 (100\%) (13,36,55-58) and even DMSO $(100 \%)(11,42,59,60)$ as vehicles to assess the neurotoxicity of new antiepileptic drug candidates administered intraperitoneally, using the rotarod test. In these studies, the time-points for the evaluation of the motor impairment were coincident with two of the points of our study $(0.5$ and/or $4 \mathrm{~h}$ ). In fact, the findings herein reported call into question the results obtained in the referred studies whenever the vehicles used have themselves a relevant neuromotor toxicity. Hence, it is clear that it is always necessary to evaluate the vehicles alone before the experiments with the compounds of interest in order to avoid possible confounding variables that influence the validity of the results.

\section{CONCLUSION}

The solubility of new chemical entities is one of the crucial parameters that has to be taken into account since the earliest steps of programs of discovery and development of new drug candidates. In fact, compounds with insufficient solubility not only can be problematic in the interpretation of results, but also can lead to additional costs. Thus, the selection of appropriate vehicles to incorporate the compounds of interest is of paramount importance to have confidence in the experimental results obtained. In this study the motor impairment (a surrogate of neurotoxicity or minimal neurological deficit) provoked by several vehicles was assessed through the rotarod performance test. As expected, all the aqueous vehicles $(\mathrm{NaCl} 0.9 \%$ and $\mathrm{CMC} 0.5 \%$ with and without DMSO) showed to be safe in this test. However, other frequently employed vehicles, such as DMSO, PEG-400 and PG induced marked neuromotor deficit in mice. This means that relevant misrepresentations of the experimental results could occur by using these vehicles to administer poorly soluble compounds to be evaluated in the rotarod performance test. In this way, this study brings further important data on the neuromotor deficit/neurotoxicity of some commonly used administration/delivery vehicles. Accordingly, this work appeals to a special attention in the selection of drug vehicles, in order to avoid the impact of such confounding variables on the accuracy of the results and in subsequent decision-making processes.

\section{CONFLICT OF INTEREST}

The authors declare no conflict of interest.

\section{ACKNOWLEDGMENTS}

The authors are grateful to Fundação para a Ciência e a Tecnologia (Lisbon, Portugal) for the $\mathrm{PhD}$ fellowship of Mariana Matias (SFRH/BD/85279/2012), involving the POPHQREN, which is co-funded by FSE and MEC. The authors also acknowledge the support provided by FEDER funds through the POCI - COMPETE 2020 - Operational Programme Competitiveness and Internationalisation in Axis I - Strengthening research, technological development and innovation (Project No. 007491) and National Funds by FCT - Foundation for Science and Technology (Project UID/Multi/00709/2013).

\section{REFERENCES}

1. Caron G, Ermondi G. Updating molecular properties during early drug discovery. Drug Discov Today, 2017; 22:835-840.

2. Di L, Fish PV, Mano T. Bridging solubility between drug discovery and development. Drug Discov Today, 2012; 17:486-495.

3. Stegemann S, Leveiller F, Franchi D, de Jong H, Lindén $\mathrm{H}$. When poor solubility becomes an issue: From early stage to proof of concept. Eur J Pharm Sci, 2007; 31:249-261.

4. Banfor PN, Gintant GA, Lipari JM, Zocharski PD. A novel intravenous vehicle for preclinical cardiovascular screening of small molecule drug candidates in rat. J Pharmacol Toxicol Methods, 
2016; 82:62-67.

5. Gad S, Cassidy CD, Aubert N, Spainhour B, Robbe H. Nonclinical Vehicle Use in Studies by Multiple Routes in Multiple Species. Int J Toxicol, 2006; 25:499-521.

6. Larsen J, Gasser K, Hahin R. An Analysis of Dimethylsulfoxide-Induced Action Potential Block: A Comparative Study of DMSO and Other Aliphatic Water Soluble Solutes. Toxicol Appl Pharmacol, 1996; 140:296-314.

7. Galvao J, Davis B, Tilley M, Normando E, Duchen MR, Cordeiro MF. Unexpected low-dose toxicity of the universal solvent DMSO. FASEB J, 2014; 28(3):1317-1330.

8. Santos NC, Figueira-Coelho J, Martins-Silva J, Saldanha C. Multidisciplinary utilization of dimethyl sulfoxide: pharmacological, cellular, and molecular aspects. Biochem Pharmacol, 2003; 65(7):1035-1041.

9. Wang X, Cattaneo F, Ryno L, Hulleman J, Reixach N, Buxbaum JN. The systemic amyloid precursor transthyretin (TTR) behaves as a neuronal stress protein regulated by HSF1 in SH-SY5Y human neuroblastoma cells and APP23 Alzheimer's disease model mice. J Neurosci, 2014; 34:72537265 .

10. Li S, Shen C, Guo W, Zhang X, Liu S, Liang F, et al. Synthesis and neuroprotective action of xyloketal derivatives in Parkinson's disease models. Mar Drugs, 2013; 11:5159-5189.

11. Mozaffari S, Ghasemi S, Baher H, Khademi H, Amini M, Sakhteman A, et al. Synthesis and evaluation of some novel methylene-bridged aryl semicarbazones as potential anticonvulsant agents. Med Chem Res, 2012; 21:3797-3808.

12. Auta J, Kadriu B, Giusti P, Costa E, Guidotti A. Anticonvulsant, anxiolytic, and non-sedating actions of imidazenil and other imidazobenzodiazepine carboxamide derivatives. Pharmacol Biochem Behav, 2010; 95:383-389.

13. Amir M, Ali I, Hassan MZ. Design and synthesis of some new quinazolin-4- $(3 \mathrm{H})$-ones as anticonvulsant and antidepressant agents. Arch Pharm Res, 2013; 36:61-68.

14. Goodfellow VS, Loweth CJ, Ravula SB, Wiemann T, Nguyen T, Xu Y, et al. Discovery, synthesis, and characterization of an orally bioavailable, brain penetrant inhibitor of mixed lineage kinase 3 . J Med Chem, 2013; 56:8032-8048.

15. Vafaeezadeh M, Hashemi MM. Polyethylene glycol (PEG) as a green solvent for carbon-carbon bond formation reactions. J Mol Liq, 2015; 207:7379.

16. Abbasi M, Martinez F, Jouyban A. Prediction of deferiprone solubility in aqueous mixtures of ethylene glycol, propylene glycol and polyethylene glycol 400 at various temperatures. J Mol Liq, 2014; 197:171-175.

17. Thore SN, Gupta S V., Baheti KG. Synthesis and Pharmacological Evaluation of Novel Triazolo [4,3-a] tetrahydrobenzo (b) thieno [3,2-e]
pyrimidine-5(4H)-ones. J Heterocycl Chem, 2015; 52:142-149.

18. Bao D, Chen M, Wang H, Wang J, Liu C, Sun R. Preparation and characterization of double crosslinked hydrogel films from carboxymethylchitosan and carboxymethylcellulose. Carbohydr Polym, 2014; 110:113-120.

19. Yang HN, Park JS, Jeon SY, Park KH. Carboxymethylcellulose (CMC) formed nanogels with branched poly(ethyleneimine) (bPEI) for inhibition of cytotoxicity in human MSCs as a gene delivery vehicles. Carbohydr Polym, 2015; 122:265-275.

20. Amenta PS, Jallo JI, Tuma RF, Elliott MB. A cannabinoid type 2 receptor agonist attenuates blood-brain barrier damage and neurodegeneration in a murine model of traumatic brain injury. $\mathrm{J}$ Neurosci Res, 2012; 90:2293-2305.

21. Cao X, Chen Y, Zhang Y, Lan Y, Zhang J, Xu X, et al. Synthesis and biological evaluation of novel sigmal receptor ligands for treating neuropathic pain: 6-Hydroxypyridazinones. J Med Chem, 2016; 59:2942-2961.

22. Harada A, Suzuki K, Kimura H. TAK-063, a novel phosphodiesterase 10A inhibitor, protects from striatal neurodegeneration and ameliorates behavioral deficits in the R6/2 mouse model of Huntington's disease. J Pharmacol Exp Ther, 2017; 360:75-83.

23. Torregrosa R, Yang X-F, Dustrude ET, Cummins TR, Khanna R, Kohn H. Chimeric derivatives of functionalized amino acids and a-aminoamides: Compounds with anticonvulsant activity in seizure models and inhibitory actions on central, peripheral, and cardiac isoforms of voltage-gated sodium channels. Bioorg Med Chem, 2015; 23:3655-3666.

24. Zhang L-M, Zhao N, Guo W-Z, Jin Z-L, Qiu Z-K, Chen H-X, et al. Antidepressant-like and anxiolytic-like effects of YL-IPA08, a potent ligand for the translocator protein $(18 \mathrm{kDa})$. Neuropharmacology, 2014; 81:116-125.

25. Hasebe M, Matsumoto I, Imagawa T, Uehara M. Effects of an anti-thyroid drug, methimazole, administration to rat dams on the cerebellar cortex development in their pups. Int J Devl Neurosci, 2008; 26:409-414.

26. Harford-Wright E, Thornton E, Vink R. Angiotensin-converting enzyme (ACE) inhibitors exacerbate histological damage and motor deficits after experimental traumatic brain injury. Neurosci Lett, 2010; 481:26-29.

27. Bagal S, Bungay P. Restricting CNS penetration of drugs to minimise adverse events: Role of drug transporters. Drug Discov Today Technol, 2014; 12:e79-85.

28. Kikuchi R, de Morais SM, Kalvass JC. In vitro Pglycoprotein efflux ratio can predict the in vivo brain penetration regardless of biopharmaceutics drug disposition classification system class. Drug 
Metab Dispos, 2013; 41:2012-2017.

29. Matias M, Campos G, Silvestre S, Falcão A, Alves G. Early preclinical evaluation of dihydropyrimidin(thi)ones as potential anticonvulsant drug candidates. Eur J Pharm Sci, 2017; 102:264-274.

30. Fortuna A, Alves G, Falcão A, Soares-Da-Silva P. Evaluation of the permeability and P-glycoprotein efflux of carbamazepine and several derivatives across mouse small intestine by the Ussing chamber technique. Epilepsia, 2012; 53:529-538.

31. Fortuna A, Alves G, Soares-da-Silva P, Falcão A. Pharmacokinetics, brain distribution and plasma protein binding of carbamazepine and nine derivatives: New set of data for predictive in silico ADME models. Epilepsy Res, 2013; 107:37-50.

32. Alves G, Figueiredo I, Castel-Branco M, Lourenço N, Falcão A, Caramona $M$, et al. Disposition of eslicarbazepine acetate in the mouse after oral administration. Fundam Clin Pharmacol, 2008; 22:529-536.

33. Castel-Branco M, Lebre V, Falcão A, Figueiredo I, Caramona M. Relationship between plasma and brain levels and the anticonvulsant effect of lamotrigine in rats. Eur J Pharmacol, 2003; 482:163-168.

34. Castel-Branco MM, Figueiredo I V., Falcão AC, Macedo TRA, Caramona MM. Influence of administration vehicles and drug formulations on the pharmacokinetic profile of lamotrigine in rats. Fundam Clin Pharmacol, 2002; 16:331-336.

35. Kshirsagar A, Toraskar MP, Kulkarni VM, Dhanashire S, Kadam V. Microwave assisted synthesis of potential anti infective and anticonvulsant thiosemicarbazones. Int J ChemTech Res, 2009; 1:696-701.

36. Kumar D, Sharma VK, Kumar R, Singh T, Singh $\mathrm{H}$, Singh AD, et al. Design, synthesis and anticonvulsant activity of some new 5,7dibromoisatin semicarbazone derivatives. EXCLI J, 2013; 12:628-640.

37. Pandeya SN, Yogeeswari P, Stables JP. Synthesis and anticonvulsant activity of 4-bromophenyl substituted aryl semicarbazones. Eur J Med Chem, 2000; 35:879-886.

38. Stella VJ, Nti-Addae KW. Prodrug strategies to overcome poor water solubility. Adv Drug Deliv Rev, 2007; 59:677-694.

39. Loh ZH, Samanta AK, Sia Heng PW. Overview of milling techniques for improving the solubility of poorly water-soluble drugs. Asian J Pharm Sci, 2014; 10:255-274.

40. Elder D, Holm R. Aqueous solubility: Simple predictive methods (in silico, in vitro and biorelevant approaches). Int J Pharm, 2013; 453:3-11.

41. Yuan C, Gao J, Guo J, Bai L, Marshall C, Cai Z, et al. Dimethyl sulfoxide damages mitochondrial integrity and membrane potential in cultured astrocytes. PLoS One, 2014; 9:e107447.

42. Dawood KM, Abdel-Gawad H, Rageb EA, Ellithey M, Mohamed HA. Synthesis, anticonvulsant, and anti-inflammatory evaluation of some new benzotriazole and benzofuran-based heterocycles. Bioorg Med Chem, 2006; 14:3672-3680.

43. Waybright TJ, Britt JR, McCloud TG. Overcoming Problems of Compound Storage in DMSO: Solvent and Process Alternatives. J Biomol Screen, 2009; 14:708-715.

44. Rammler D. Biological Implications of Dmso Based on a Review of Its Chemical Properties. Ann New York Acad, 1967; (40):13-23.

45. Authier N, Dupuis E, Kwasiborski A, Eschalier A, Coudore F. Behavioural assessment of dimethylsulfoxide neurotoxicity in rats. Toxicol Lett, 2002; 132:117-121.

46. Bakar B, Kose EA, Sonal S, Alhan A, Kilinc K, Keskil IS. Evaluation of the neurotoxicity of DMSO infused into the carotid artery of rat. Injury, 2012; 43:315-322.

47. Julien C, Marcouiller F, Bretteville A, El Khoury NB, Baillargeon J, Hébert SS, et al. Dimethyl sulfoxide induces both direct and indirect tau hyperphosphorylation. PLoS One, 2012; 7:e40020.

48. Lu C, Mattson MP. Dimethyl sulfoxide suppresses NMDA- and AMPA-induced ion currents and calcium influx and protects against excitotoxic death in hippocampal neurons. Exp Neurol, 2001; 170:180-185.

49. Cavaletti G, Oggioni N, Sala F, Pezzoni G, Cavalletti E, Marmiroli P, et al. Effect on the peripheral nervous system of systemically administered dimethylsulfoxide in the rat: a neurophysiological and pathological study. Toxicol Lett, 2000; 118:103-107.

50. Hanslick JL, Lau K, Noguchi KK, Olney JW, Zorumski CF, Mennerick S, et al. Dimethyl sulfoxide (DMSO) produces widespread apoptosis in the developing central nervous system. Neurobiol Dis, 2009; 34:1-10.

51. Lau K, Swiney BS, Reeves N, Noguchi KK, Farber NB. Propylene glycol produces excessive apoptosis in the developing mouse brain, alone and in combination with phenobarbital. Pediatr Res, 2012; 71:54-62.

52. Colucci M, Maione F, Bonito MC, Piscopo A, Di Giannuario A, Pieretti S. New insights of dimethyl sulphoxide effects (DMSO) on experimental in vivo models of nociception and inflammation. Pharmacol Res, 2008; 57:419-425.

53. Zar T, Graeber C, Perazella MA. Recognition, treatment, and prevention of propylene glycol toxicity. Semin Dial, 2007; 20:217-219.

54. Thackaberry EA, Wang X, Schweiger M, Messick K, Valle N, Dean B, et al. Solvent-based formulations for intravenous mouse pharmacokinetic studies: tolerability and recommended solvent dose limits. Xenobiotica, 2014; 44:235-241.

55. Kashaw SK, Kashaw V, Mishra P, Jain NK, Stables JP. Synthesis, anticonvulsant and CNS depressant activity of some new bioactive 1-(4-substitutedphenyl)-3-(4-oxo-2-phenyl/ethyl-4H-quinazolin-3- 
yl)-urea. Eur J Med Chem, 2009; 44:4335-4343.

56. Alam O, Mullick P, Verma SP, Gilani SJ, Khan SA, Siddiqui N, et al. Synthesis, anticonvulsant and toxicity screening of newer pyrimidine semicarbazone derivatives. Eur J Med Chem, 2010; 45:2467-2472.

57. Cui L-J, Xie Z-F, Piao H-R, Li G, Chai K-Y, Quan Z-S. Synthesis and Anticonvulsant Activity of 1Substituted-7-Benzyloxy-4,5- dihydro$[1,2,4]$ triazolo[4,3-a]quinoline. Biol Pharm Bull, 2005; 28:1216-1220.

58. Partap S, Akhtar MJ, Yar MS, Hassan MZ, Siddiqui AA. Pyridazinone hybrids: Design, synthesis and evaluation as potential anticonvulsant agents. Bioorg Chem, 2018; 77:74-83.

59. Zhang W Bin, Han RB, Zhang W, Jiang RS, Piao FY. Synthesis and anticonvulsant activity of 8alkoxy-5,6-dihydro-4Hbenzo[f][1,2,4]triazolo[4,3a]azepine derivatives. Med Chem Res, 2012; 21:2587-2594.

60. Xie C, Tang L-M, Li F-N, Guan L-P, Pan C-Y, Wang S-H. Structure-based design, synthesis, and anticonvulsant activity of isatin-1-Nphenylacetamide derivatives. Med Chem Res, 2014; 23:2161-2168. 